\title{
Cambios vegetacionales antropogénicos en el Cerro Catedral (Río Negro, Argentina)
}

\author{
Human impact and vegetation change in Mt. Catedral (Río Negro, Argentina)
}

\author{
ALEJANDRO DEZZOTTI, ${ }^{1}$ LUIS SANCHOLUZ, ${ }^{2}$ MARTIN NAUMANN ${ }^{3}$
}

1 Asentamiento Universitario San Martín de los Andes. email: dezzotti@infovia.com.ar.

${ }^{2}$ Centro Regional Universitario Bariloche, Universidad Nacional del Comahue, Pasaje de la Paz 235, Q8370AQA San Martín de los Andes, Argentina.

${ }^{3}$ Fundación Proyecto Andino, San Carlos de Bariloche, Argentina.

\section{SUMMARY}

Skiing started around 65 years ago on the northern sector of the eastern slope of Mt. Catedral $\left(41^{\circ} 10^{\prime} \mathrm{S}, 71^{\circ} 30^{\prime} \mathrm{W}\right.$, $2,388 \mathrm{~m}$ ), and currently the mountain hosts the most important winter sports center in South America. Current and potential vegetation types were compared, based on historical vegetation maps and photographs, topographic and climatic maps, recent aerial photographs and the forest structure. The modern vegetation is made up of: i) meadows (10.7\% of the study area); ii) Austrocedrus chilensis forests (2.2\%); iii) Nothofagus antarctica and Chusquea culeou shrublands (29.7\%); iv) Nothofagus pumilio forests and shrublands (26.7\%); and v) alpine vegetation (19.5\%). Although large-scale disturbances occurred before 1937, the expansion of the ski center caused extensive clear-felling for construction of ski runs, slopes, roads, means of transport, and amenities. Around $9 \%$ of the originally forested land was completely replaced by secondary, anthropogenic areas (i.e., urban and non-wooded).The current stand structure reflects different disturbance regimes and forest recovery. The deforestation of this fragile ecosystem has resulted in landscape, soil, and biota degradation.

Key words: deforestation, assessment, Nothofagus pumilio, skiing, Patagonia.

\section{RESUMEN}

En el sector septentrional de la ladera Este del cerro Catedral (41 $\left.{ }^{\circ} 10^{\prime} \mathrm{S}, 71^{\circ} 30^{\prime} \mathrm{O}, 2.388 \mathrm{~m}\right)$ la práctica del esquí comenzó hace alrededor de 65 años y en la actualidad se desarrolla el centro de deportes invernales más importante de Sudamérica. Se compararon los tipos de vegetación actual y potencial a partir de mapas y fotografías históricas, fotografías aéreas actuales, mapas climáticos y topográficos y la estructura del bosque. La vegetación actual está representada por i) la pradera (10,7\% del área de estudio), ii) el bosque de Austrocedrus chilensis (2,2\%), iii) el matorral de Nothofagus antarctica y Chusquea culeou (29,7\%), iv), el bosque y matorral de Nothofagus pumilio (26,7\%) y v) la vegetación altoandina (19,5\%). Aunque antes de 1937 ocurrieron disturbios masivos de gran escala, el desarrollo del centro de deportes implicó talas rasas extensivas para la construcción y el emplazamiento de pistas de esquí, caminos, medios de transporte e infraestructura. El $9 \%$ del área boscosa se reemplazó por áreas urbanizadas y con vegetación secundaria no arbórea. La estructura actual del bosque refleja diferentes regímenes de disturbios y la posterior recuperación de la vegetación. La deforestación de este frágil ecosistema provocó una degradación del paisaje, el suelo y la biota.

Palabras clave: deforestación, evaluación, Nothofagus pumilio, esquí, Norpatagonia. 
BOSQUE 25(3): 63-71, 2004

Cambios vegetacionales antropogénicos en el Cerro Catedral (Río Negro, Argentina)

\section{INTRODUCCION}

Las montañas constituyen ambientes intrínsecamente frágiles debido a las bajas temperaturas, las altas precipitaciones, la fuerte intensidad y frecuencia de los vientos y las pendientes pronunciadas con longitudes extensas. En estas áreas la recreación especialmente asociada al esquí y snowboard representa un riesgo ambiental adicional por la alta concentración de visitantes en cortos períodos de tiempo, la densa red de pistas y medios de elevación y la producción artificial de nieve (1). En general, los centros de deportes invernales se localizan en terrenos boscosos y el esquí se practica sobre talas rasas permanentes a lo largo de pendientes acentuadas. Tradicionalmente, el diseño e implementación de estos emprendimientos sólo se basó en consideraciones técnicas y socioeconómicas, omitiendo el análisis de la restricción y sensibilidad del ambiente físico y biológico. Sin embargo, se están desarrollando técnicas analíticas específicas para identificar y mitigar los múltiples efectos ambientales negativos de las actividades masivas de montaña sobre el microclima, el suelo, la biota, el relieve, la hidrología y los aspectos estéticos del paisaje (1, 2, 3).

El centro de deportes invernales Antonio Lynch se localiza sobre el sector septentrional de la ladera Este del cerro Catedral a los $41^{\circ} 13^{\prime} \mathrm{S}$. y $71^{\circ} 30^{\prime} \mathrm{O}$ y forma parte del egido de San Carlos de Bariloche (Argentina) (figura 1). Aunque en la actualidad se considera el centro más importante de Sudamérica, en la Argentina el esquí comenzó en otras áreas de montaña. La primera carrera de esquí se llevó a cabo en 1932 en el cerro Otto y también se practicaba este deporte en 1935 en el cerro Ventana, ambos en la misma área de San Carlos de Bariloche (4). En el cerro Catedral recién en la temporada de invierno de 1937 se desarrollaron las primeras competencias, en 1938 se trazaron los primeros caminos, en 1943 se construyeron el primer refugio de montaña (el actual Refugio Lynch) y el primer Sky-lift, y en 1953 se inauguró el cable-carril $(4,5)$. En la actualidad el centro de deportes comprende 500 ha y 32 medios de elevación que cubren $18.360 \mathrm{~m}$ con una capacidad de transporte de 24.500 ind $\mathrm{h}^{-1}$. En el presente trabajo se analiza la distribución de la vegetación actual y se la compara con la vegetación potencial que se desarrollaría en ausencia de

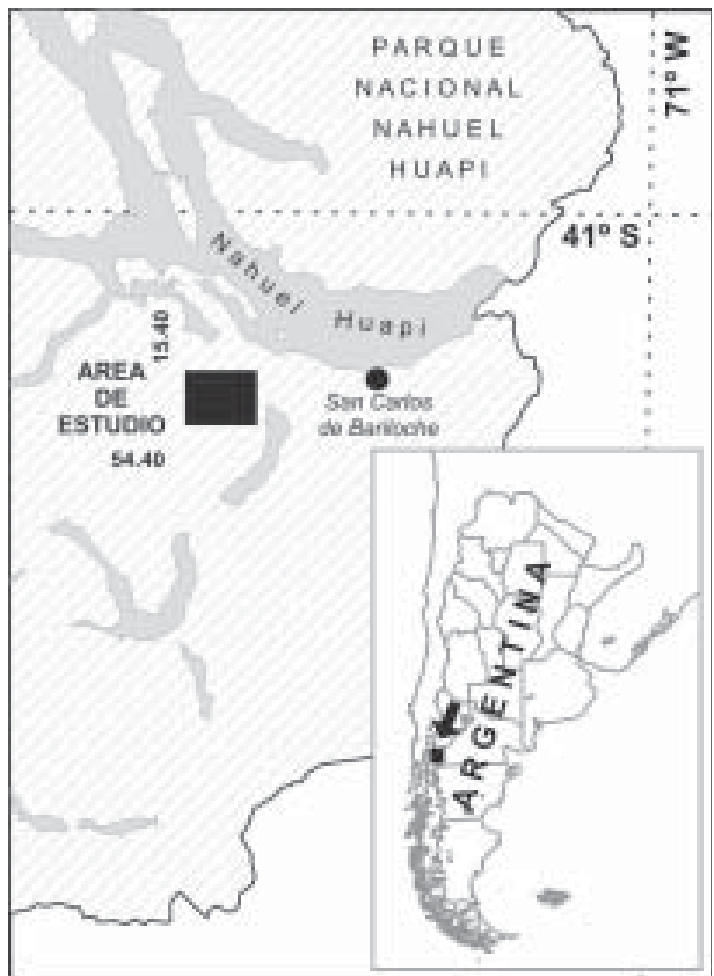

Figura 1. Localización del área de estudio (en negro) ampliada en la figura 2 .

Location of study area (shaded) enlarge in figure 2.

disturbios antropogénicos asociados al desarrollo del centro de deportes de invierno en el sector septentrional de la ladera Este del cerro Catedral.

\section{MATERIAL Y METODOS}

El clima del cerro Catedral es templado frío y húmedo, estacional y ventoso, y se clasifica como perhúmedo microtermal (6). A medida que aumenta la altitud la temperatura media disminuye aproximadamente $0,5^{\circ} \mathrm{C}$ cada $100 \mathrm{~m}$, mientras que la amplitud térmica día-noche y veranoinvierno, el período con heladas y con cobertura de nieve, la frecuencia y la intensidad del viento y la precipitación en forma de nieve aumentan $(7,8)$ (cuadro 1). Las formaciones geológicas aflorantes del cerro Catedral son el Basamento Cristalino y la Formación Ventana (9). La primera es posiblemente Precámbrica, se ubica por encima de los $1.500 \mathrm{~m}$ y está formada por rocas ígneas en contacto intrusivo con rocas metamór- 


\section{CUADRO 1}

Datos climáticos de San Carlos de Bariloche $\left(41^{\circ} 09^{\prime} \mathrm{S}, 71^{\circ} 18^{\prime} \mathrm{O}, 760 \mathrm{~m}\right)$

y Refugio Lynch en el cerro Catedral

(41 $\left.{ }^{\circ} 10^{\prime} \mathrm{S}, 71^{\circ} 20^{\prime} \mathrm{O}, 1.950 \mathrm{~m}\right)(7,8)$.

Climate data from San Carlos de Bariloche

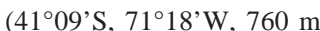

and Refugio Lynch in Mt. Catedral

$\left(41^{\circ} 10^{\prime} \mathrm{S}, 71^{\circ} 20^{\prime} \mathrm{W}, 1,950 \mathrm{~m}\right)(7,8)$

\begin{tabular}{|c|c|c|}
\hline Variable & $\begin{array}{l}\text { San Carlos } \\
\text { de Bariloche }\end{array}$ & $\begin{array}{c}\text { Refugio } \\
\text { Lynch }\end{array}$ \\
\hline \multicolumn{3}{|l|}{ Cerro Catedral } \\
\hline Temperatura $\left({ }^{\circ} \mathrm{C}\right)$ & 8,4 & 2,4 \\
\hline Precipitación $\left(\mathrm{mm}\right.$ año $\left.{ }^{-1}\right)$ & 1.097 & 1.467 \\
\hline Helada (días año-1) & 76 & 194 \\
\hline Viento $\left(\mathrm{km} \mathrm{h}^{-1}\right)$ & 18 & 30 \\
\hline Nieve (días año ${ }^{-1}$ ) & 20 & 98 \\
\hline
\end{tabular}

ficas recubiertas por detritos. La segunda está constituida por andesitas, brechas volcánicas, wackes y tufitas provenientes del Eoceno que están en contacto tectónico con la anterior. El paisaje está modelado por la glaciación y el vulcanismo del Cuaternario (10). El vulcanismo es predominantemente basáltico y produce arenas y cenizas volcánicas que constituyen el material parental de los suelos desarrollados en el área (11). El suelo se clasifica como Andosol (12) y constituye un manto continuo sólo en los sectores de menor altitud. La vegetación pertenece al Distrito del Bosque Caducifolio de los Bosques Subantárticos (13).

El mapa de vegetación del sector septentrional de la ladera Este del cerro Catedral (figura 2) se desarrolló mediante la interpretación de las fotografías aéreas OJ 316 -2.689 y 2.690, 1:60.000, ampliadas a 1:12.000 (Prefectura Naval Argentina 1981) y del grupo fotográfico I, corridas 7 y 8 , 1:20.000 (Fuerza Aérea Argentina 1994). La correspondencia entre la vegetación observada en las fotografías y la presente en el área de estudio se constató en el terreno. Se identificaron las especies de plantas más frecuentes en cada unidad de vegetación. El mapa de vegetación potencial del área de estudio (figura 3) se basó en la clasificación de tipos de hábitat de Daubenmire $(14,15)$ que establece que aquellos sitios capaces de desarrollar potencialmente una comunidad de plantas similar durante los estadios estables de la sucesión se reúnen en la misma categoría. La vegetación potencial se interpretó como la que se desarrollaría en ausencia de la actividad del centro de deportes invernales y se la estimó conociendo los rangos de tolerancia ecológica de las especies dominantes, principalmente con relación a variables climáticas y topográficas, utilizando fotografías aéreas y mapas de precipitación (7) y topográficos del Instituto Geográfico Militar (escala 1:50.000, equidistancia $25 \mathrm{~m}$ ). La vegetación actual se la comparó con la del sector meridional de la ladera Este del cerro Catedral, donde las condiciones físicas son similares a las que ocurren en el área de estudio, aunque allí no se desarrollan actividades humanas degradativas asociadas a los deportes masivos de montaña.

La información cartográfica se procesó con un Sistema de Información Geográfico sobre un mapa georreferenciado. Las características de la vegetación en períodos previos al comienzo del desarrollo del centro de deportes de invierno se obtuvieron consultando información histórica. Willis (16) reveló las comunidades vegetales a principios de siglo y Nöbl (17) produjo un archivo fotográfico durante el diseño de emplazamiento de las primeras pistas de esquí, los medios de elevación y las obras de infraestructura del cerro Catedral. Para caracterizar la vegetación dominada por Nothofagus pumilio (Poeppig et Endl.) Krasser (Nothofagaceae) se instalaron seis parcelas circulares de muestreo de $500 \mathrm{~m}^{2}$ cada una y se midieron los diámetros a la altura del pecho (DAP, con cinta diamétrica) a todos los árboles $4 \mathrm{~cm}$ DAP presentes en cada parcela. Se estimaron la abundancia y el tamaño de los árboles muertos en pie. El área basal de cada individuo se asumió como equivalente a la de un círculo de la misma circunferencia del árbol. Se midió la altura total de cuatro árboles por parcela, seleccionados al azar utilizando un clinómetro. Para estimar la altura total de cada árbol se registraron los ángulos a la base y al extremo superior, y la distancia horizontal desde el observador hasta el árbol.

\section{RESULTADOS Y DISCUSION}

La vegetación zonal del área de estudio (cuyas características resultan básicamente de la influencia del clima) estuvo representada por i) la 


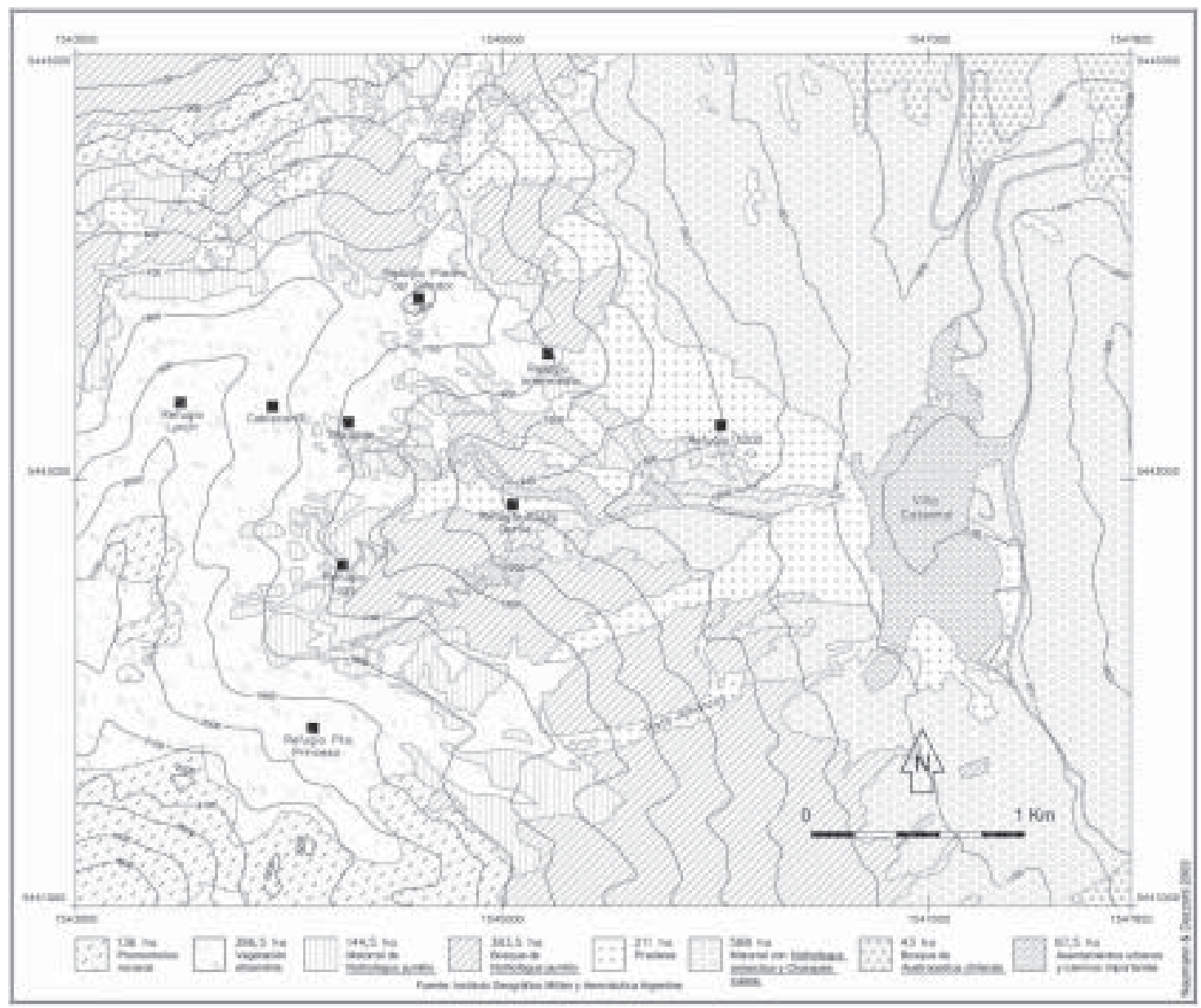

Figura 2. Localización de los tipos de vegetación actual y la infraestructura del sector septentrional de la ladera Este del cerro Catedral.

Location of current vegetation types and infrastructure within the northern sector of the eastern slope of Mt. Catedral.

vegetación altoandina, ii) el bosque y matorral de Nothofagus pumilio, iii) el matorral de Nothofagus antarctica ((Forst.) Oerst.) y Chusquea culeou (Desv., Gramineae), iv) el bosque de Austrocedrus chilensis ((Don) Florin et Boutleje, Cupressaceae) y v) la pradera. La vegetación intrazonal (cuyas características resultan básicamente de la influencia de factores locales) estuvo representada por el mallín de altura. La vegetación altoandina se extendió desde los 1.850 y hasta los $2.200 \mathrm{~m}$ y ocupó el $19,5 \%$ del área de estudio (figura 2, cuadro 2). La alta frecuencia e intensidad del viento, las grandes variaciones y extremos de temperatura, la gruesa capa de nieve que cubre el suelo y la fuerte incidencia solar imponen serias restricciones para el desarrollo de este tipo de vegetación. Las plantas se encontraron en forma dispersa, cubrien- do hasta el $10 \%$ de la superficie del suelo. Las formas de crecimiento dominantes fueron herbáceas perennes y sufrútices, en cojín y rastreras. Las especies más frecuentes fueron Acaena leptacantha Philippi (Rosaceae), Loasa nana Philippi (Loasaceae), Luzula chilensis Nees et Meyen (Juncaceae), Perezia pilifera (Don) Hooker et Arnott, Senecio argyreus Philippi (Compositae) y Viola cotyledon Gingins in DC (Violaceae).

El bosque y matorral de Nothofagus pumilio representó el 26,7\% del área de estudio (figuras 2, cuadro 2). Entre los 1.400 y $1.850 \mathrm{~m}$ esta especie sólo alcanzó los $2 \mathrm{~m}$ de altura y formó una comunidad densa y discontinua, con escaso desarrollo de vegetación herbácea. Las especies que circundaron estos matorrales fueron Pernettya pumilia Hooker (Ericaceae), Poa tristigmata 


\section{CUADRO 2}

Superficie urbanizada y de la vegetación actual y potencial en el sector septentrional de la ladera Este del cerro Catedral.

Current and potential vegetation types and urbanized areas within the northern sector of the eastern slope of Mt. Catedral.

\begin{tabular}{|l|r|r|r|r|r|r|}
\hline \multirow{2}{*}{ Unidad } & \multicolumn{3}{|c|}{ Superficie } & \multicolumn{2}{c|}{ Cambio } \\
\cline { 2 - 7 } & \multicolumn{2}{|c|}{ Actual (A) } & \multicolumn{1}{|c|}{ Potencial (P) } & \multicolumn{1}{c|}{ A-P } & A-P/P \\
\cline { 2 - 7 } & ha & \multicolumn{1}{|c|}{ ha } & $\%$ & ha & $\%$ \\
\hline \multirow{2}{*}{ Promontorios rocosos } & 136,0 & 6,9 & 136,0 & 6,9 & - & - \\
Vegetación altoandina & 386,5 & 19,5 & 343,0 & 17,3 & 43,5 & 12,7 \\
Matorral de Nothofagus pumilio & 144,5 & 7,3 & 176,5 & 8,9 & $-32,0$ & $-18,1$ \\
Bosque de Nothofagus pumilio & 383,5 & 19,4 & 473,0 & 23,9 & $-89,5$ & $-18,9$ \\
Matorral de Nothofagus antarctica & & & & & & \\
y Chusquea culeou & 588,0 & 29,7 & 650,0 & 32,8 & $-62,0$ & $-9,5$ \\
Bosque de Austrocedrus chilensis & 43,0 & 2,2 & 43,0 & 2,2 & - & - \\
Pradera & 211,0 & 10,7 & 158,5 & 8,0 & 52,5 & 33,1 \\
Asentamiento urbano y caminos & 87,5 & 4,4 & - & - & 87,5 & \\
Total & 1.980 & 100 & 1.980 & 100 & & \\
\hline
\end{tabular}

Desvaux ap. Gay (Gramineae), Quinchamalium chilense Lam. (Santalaceae), Discaria serratifolia (G. Don) Tortosa (Rhamnaceae), Berberis empetrifolia Lam. (Berberidaceae) y Ribes magellanicum Poiret (Saxifragaceae). Entre los 1.200 y $1.400 \mathrm{~m}$ se desarrolló el bosque alto constituido por Nothofagus pumilio con las especies arbustivas Aristotelia chilensis (Mol.) Stuntz (Eleocarpaceae), Berberis serrato-dentata Lechl. y Chusquea culeou, y las herbáceas Adenocaulon chilense Less. (Compositae), Alstroemeria aurea Graham. (Amarilidaceae), Osmorrhiza chilensis Hooker et Arn. (Umbeliferae) y Vicia nigricans Hooker et Arnott (Leguminosae). Como consecuencia de los modos de regeneración catastrófico a gran escala y de claros a escala espacial más reducida, Nothofagus pumilio adopta una estructura en mosaico constituido por grupos de árboles coetáneos de diferentes edades que ocupan superficies variables (18). En el cerro Catedral se encontraron rodales jóvenes con una estructura de tamaños regular (estación 2, cuadro 3, figura 4), que se habrían originado después de la ocurrencia de disturbios masivos, y rodales más maduros con una estructura de tamaños irregular conformada por individuos de todas las clases de tamaño (estación 1 , cuadro 3 , figura 4 ), que se habrían originado después de la caída de árboles maduros que produjeron claros ocupados por otras cohortes. En una especie heliófila como Nothofagus pumilio, la estructura de tallas indica el grado de coetaneidad en razón de la relación frecuentemente observada entre la edad y el DAP de los árboles (19).

El matorral de Nothofagus antarctica y Chusquea culeou se encontró entre los 1.000 y 1.200 m, ocupó el 29,7\% del área de estudio y estaba asociado a arbustos como Diostea juncea (Shaver) Miers (Verbenaceae), Lomatia hirsuta (Lam.) Diels, Embothrium coccineum Forst. et Forster (Proteaceae), Schinus patagonica (Phil.) Johnst. (Anacardiaceae), Aristotelia chilensis y Fabiana imbricata Ruiz et Pavón (Solanaceae) (figura 2, cuadro 2). A través de la expansión vegetativa, Chusquea culeou forma extensas poblaciones monogenéticas de hasta $7 \mathrm{~m}$ de altura, tanto debajo de la canopia cerrada como en claros del bosque virgen o en sitios derivados de disturbios naturales de gran escala, que causan niveles muy bajos de luminosidad inhibiendo el establecimiento y desarrollo de Nothofagus (20). En el cerro Catedral colonizó sectores deforestados, primariamente ocupados por los bosques de Nothofagus pumilio. El bosque de Austrocedrus chilensis representó una comunidad escasamente representada que cubrió el 2,2\% del área de estudio, donde se encontraron Lomatia hirsuta, 
BOSQUE 25(3): 63-71, 2004

Cambios vegetacionales antropogénicos en el Cerro Catedral (Río Negro, Argentina)

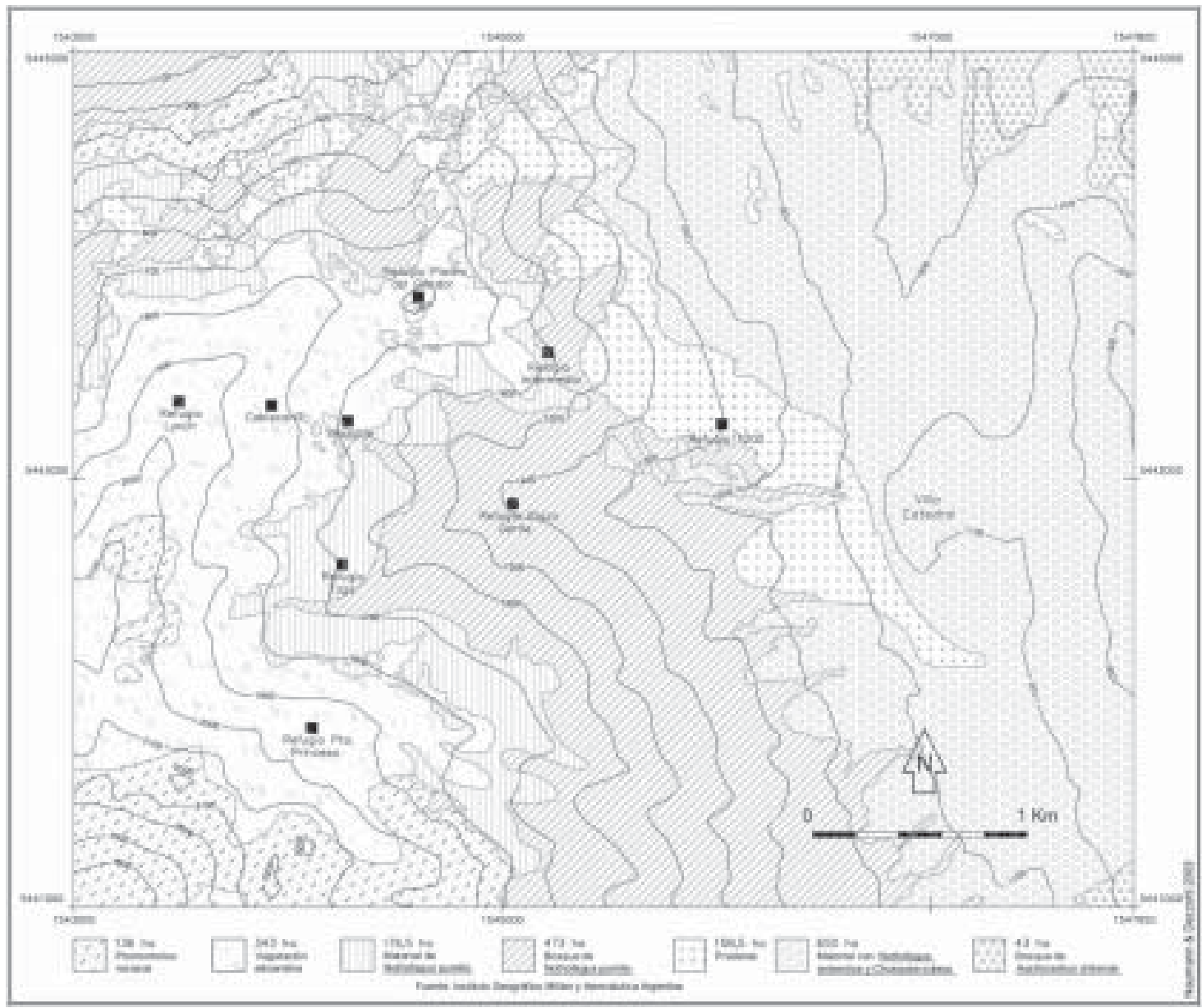

Figura 3. Localización de los tipos de vegetación potencial del sector septentrional de la ladera Este del cerro Catedral.

Location of potential vegetation types within the northern sector of the eastern slope of Mt. Catedral.

\section{CUADRO 3}

Estructura de población de Nothofagus pumilio en las estaciones 1, 2 y 6 . Population structure of Nothofagus pumilio in plots 1,2, and 6.

\begin{tabular}{|l|c|c|c|}
\hline \multirow{2}{*}{ Variable } & \multicolumn{3}{|c|}{ Estación } \\
\cline { 2 - 4 } & 1 & 2 & 6 \\
\hline Densidad total (ind ha $\left.{ }^{-1}\right)^{(a)}$ & 400 & 1.980 & 1.560 \\
Area basal $\left(\mathrm{m}^{2} \mathrm{ha}^{-1}\right)^{-1}$ & 79,3 & 72,8 & 74,8 \\
Altura (m ind $)^{-1}$ & 20 & 17 & 12 \\
Densidad renovales (ind ha $\left.^{-1}\right)^{(\mathrm{b})}$ & - & 1.485 & 1.000 \\
Densidad árboles muertos (ind ha $\left.^{-1}\right)^{(\mathrm{c})}$ & - & 700 & 840 \\
Estadio (d) & Transición & Autoraleo & Autoraleo \\
\hline
\end{tabular}

(a) Árboles 4 cm DAP. (b) Árboles de entre 4 y 19 cm DAP. (c) Árboles muertos en pie (> $1 \mathrm{~m}$ de altura). ${ }^{(d)}$ Fase del ciclo de desarrollo del bosque de acuerdo al modelo de Peet y Christensen (24) 
BOSQUE 25(3): 63-71, 2004 Cambios vegetacionales antropogénicos en el Cerro Catedral (Río Negro, Argentina)
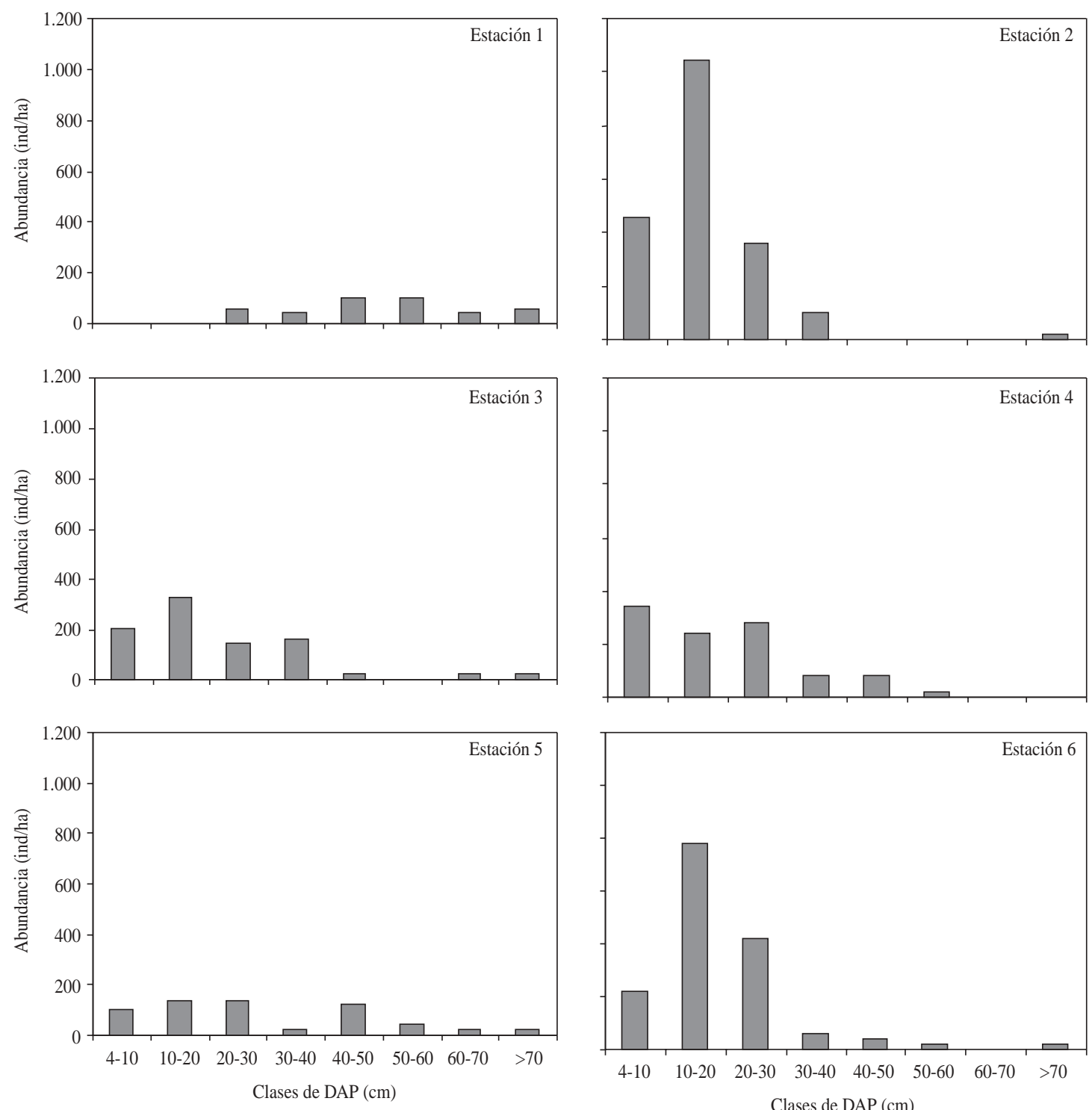

Figura 4. Distribución de las frecuencias de diámetro a la altura del pecho (DAP) de Nothofagus pumilio en las estaciones 1 a la 6 .

Diameter at breadth height (DAP) frequency distribution of Nothofagus pumilio within plots 1 to 6 .

Maytenus boaria Mol. (Celastraceae), Aristotelia chilensis, Fabiana imbricata, Schinus patagonica, Nothofagus antarctica y Alstromeria aurea (figura 2, cuadro 2). La pradera es una comunidad secundaria que se desarrolló a altitudes intermedias en las laderas expuestas hacia el norte y que representó el 10,7\% del área de estudio (figura 2, cuadro 2). Estuvo conformada por Acaena ovalifolia Ruiz et Pavón, Acaena splendens Gil. ex Hook., Anemone multifida Poiret (Ranunculaceae), Baccharis magellanica (Lam.) Pers. (Compositae), Fragaria chiloensis (L.) Duchesne (Rosaceae) y Vicia nigricans. El mallín de altura se localizó sobre planicies mal drenadas, cubriendo una superficie que por su dimensión no pudo ser cartografiada con los métodos utilizados. Las especies más frecuentes fueron Empetrum rubrum Vahl ex Willd. (Empetraceae), Ortachne brevisecta 
BOSQUE 25(3): 63-71, 2004

Cambios vegetacionales antropogénicos en el Cerro Catedral (Río Negro, Argentina)

Hitchcock (Gramineae), Calandrinia affinis Gillies (Portulacaceae), Luzula chilensis, Senecio trifurcatus (Forst. f.) Less. y Poa tristigmata.

El área de estudio estuvo frecuentemente afectada por incendios de gran escala previamente al emplazamiento del centro de deportes. Sobre la ladera Este del cerro Catedral, Willis (16) identificó en el sector septentrional (donde se emplaza el centro de esquí) áreas ocupadas por vegetación arbustiva que interpretó como quemadas recientemente, y en el sector meridional áreas ocupadas por vegetación arbustiva y arbórea que interpretó como quemadas antiguamente. El informe redactado por Nöbl (17) consta de un archivo fotográfico del cerro Catedral, donde en las fotografías de mayor escala del sector septentrional de la ladera no se observa el bosque de Nothofagus pumilio, mientras que en el sector meridional sí se observa esta comunidad. En otras fotografías a menor escala que sólo incluyen el sector septentrional de la ladera se observan árboles muertos en pie, rodeados por Chusquea culeou y renovales de Nothofagus pumilio. Arko (5) indica que en 1938 se realizaban competencias de esquí en las pistas inferiores, que estaban entonces cubiertas por un denso cañaveral de Chusquea culeou. Las fotografías y la información histórica apoyan las inferencias hechas por Willis (16) en relación con las historias diferentes de disturbios que afectaron ambos sectores de la ladera Este del cerro Catedral. Aunque en general la regeneración de Nothofagus pumilio en rodales postfuego es errática, probablemente en función de diferencias físicas y biológicas entre sitios (18), en el cerro Catedral esta especie recolonizó laderas previamente incendiadas y menos sometidas a la presión antrogénica como se infiere de la comparación entre fotografías pasadas y actuales (figura 5).

El desarrollo del centro de deportes de invierno que se llevó a cabo durante los últimos 65 años provocó modificaciones sobre la vegetación, principalmente asociados a la eliminación total del bosque y la corta selectiva de árboles en los lugares donde se emplazan las pistas de esquí, los medios de elevación y el área urbana. Las zonas previamente ocupadas por vegetación arborescente fueron reemplazadas por terrenos urbanizados o colonizadas por plantas herbáceas y arbustivas que constituyen comunidades secundarias, sucesionales tempranas, representadas principalmente por el cañaveral y la pradera. El reemplazo

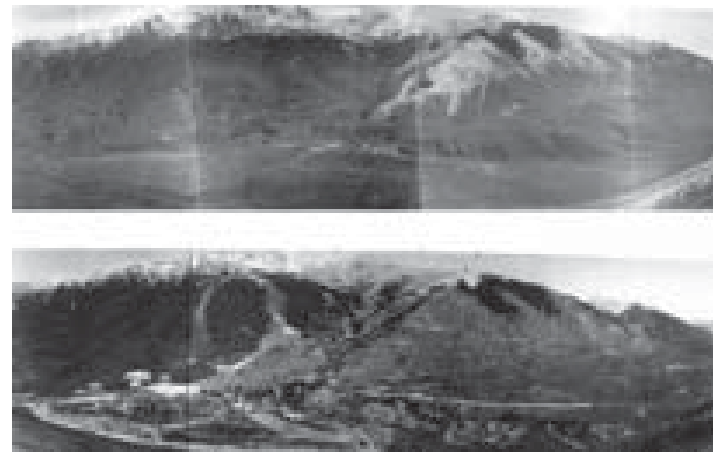

Figura 5. Fotografías del sector septentrional de la ladera Este del cerro Catedral tomadas en 1953 (arriba) y 1997 (abajo) (fotos de D. Vallmitjana).

Photographs of the northern sector of the eastern slope of Mt. Catedral taken in 1953 (above) and 1997 (bellow) (photos courtesy of D. Vallmitjana)

de la vegetación primaria por la secundaria y por el área urbana involucró alrededor de 184 ha, que representan el 9\% del área de estudio. El bosque y matorral de Nothofagus pumilio es el tipo de vegetación más afectado con una disminución aproximada de 122 ha (19\% del área potencial de este tipo de vegetación). La infraestructura y práctica del esquí perturban básicamente el piso altitudinal donde las condiciones físicas permiten el desarrollo de la vegetación, que cumple un papel de protección importante contra la erosión hídrica, eólica y gravitatoria del suelo. En general, los suelos de las regiones templadas derivados de cenizas volcánicas se consideran muy fértiles y productivos, aunque estas propiedades inherentes a menudo cambian en forma irreversible cuando la vegetación no se maneja apropiadamente (21). En el cerro Catedral la presencia extensiva de suelo desnudo, regueras, cárcavas, conos de deyección y barrancos se asocia a los cambios en la vegetación (22). Además, la desaparición de zorros (Ducysion spp, Canidae), hurones (Galictis cuja, Mustelidae), pumas (Puma concolor, Felidae) y huemules (Hippocamelus bisulcus, Cervidae) coincide con el comienzo de las actividades de esquí (23). Aunque el cerro Catedral exhibe un importantísimo valor ecológico, social y económico, las actividades humanas desarrolladas durante los últimos 65 años catalizaron una marcada disrupción ambiental, a través de la remodelización del paisaje que afectó su integridad y estabilidad intrínseca física y biológica. La reconciliación de la dimensión económica y eco- 
lógica del desarrollo sólo será posible a través de un estricto manejo conservativo para que los valores, bienes y servicios asociados a este sistema persistan a lo largo del tiempo.

\section{AGRADECIMIENTOS}

El presente trabajo se realizó con la asistencia de C. Brión, C. Ezcurra, G. Galende, D. Grigera, A. Guasp, F. Planas, J. Puntieri, A. Trejo, C. Ubeda (Universidad Nacional del Comahue), R. Marino (Ministerio de Turismo de Río Negro), A. Marcolín (Instituto Nacional de Tecnología Agropecuaria) y D. Vallmitjana. La Universidad Nacional del Comahue y Sky-World S.A. financiaron el estudio.

\section{BIBLIOGRAFIA}

(1) PFEFFER, K., T. W. J. VAN ASCH, P. A. BURROUGH A. G. FABBRI. Integration of modeling approaches in the assessment of environmental impact of ski pistes in alpine areas. Proceedings 4th International Conference on Integrating GIS and Environmental Modeling: problems, prospects and research needs, 2000, Banff, Canada.

(2) BYRNE, N. Recreation impacts in the Australian Alps. Trees and Natural Resources, 1997, vol. 39, N. 4, p. 17-19.

(3) TAPPEINER U., E. TASSER, G. TAPPEINER. Modeling vegetation patterns using natural and anthropogenic influence factors: preliminary experience with a GIS based model applied to an alpine area. Ecological Modelling, 1998, vol. 113, p. 225-237.

(4) VAllmituANA, R. 90 años de turismo en Bariloche. San Carlos de Bariloche: Asociación Empresaria Hotelera Gastronómica de Bariloche, 1993, 68 p.

(5) ARKO, V. Un pionero de Bariloche, Otto Meiling. San Carlos de Bariloche: Ediciones del Club Andino Bariloche-Imprenta Bavaria. 1991. 125 p.

(6) THORNWAITE, C. An approach toward a rationa classification of climate. Geographical Review, 1948, vol. $83, \mathrm{~N}^{\circ} 1$, p. 55-94.

(7) BARROS, V., V. CORDON, C. MOYANO, R MENDEZ, J. FORQUERA, O. PIZZIO. Cartas de precipitación de la zona oeste de las provincias de Río Negro y Neuquén. Publicación Universidad Nacional del Comahue-Centro Nacional Patagónico. Cinco Saltos, 1983, $28 \mathrm{p}$.

(8) MINETTI, J., A. CORTE. 1985. Zonificación latitudinal del clima en la zona andina y su relación con el límite inferior del hielo perenne (LIHP) y del límite inferior geocriogénico (LIG). Anales del IANIGLA (Mendoza), 1985, No 6, p. $129-144$

(9) GONZALEZ BONORINO, F. Geología del área entre San Carlos de Bariloche y Llao-Llao. San Carlos de Bariloche: Fundación Bariloche. Publicación 16. Departamento de Recursos Naturales y Energía, 1973, 55 p.

(10) MERCER, J. Late Cenozoic glacial variations in South America south of the Ecuador. In: VOGEL, J.C. Late paleoclimates of the southern hemisphere. Rotterdam: Balkema, 1984, p. 45-58.

(11) CLAPPERTON, C. Quaternary geology and geomorphology of South America. Amsterdam: Elsevier. 1993. $769 \mathrm{p}$.

(12) FAO-UNESCO soil map of the world: revised legend. Roma: Soils Bulletin 60. Food and Agriculture Organization. 1990. 119 p.

(13) CABRERA, A. L. Fitogeografía de la República Argentina. Boletín de la Sociedad Argentina de Botánica, 1971, vol. $14, \mathrm{~N}^{\circ} 1-2, \mathrm{p} .1-42$.

(14) DAUBENMIRE, R. Plant communities: a textbook of plant synecology. New York: Harper \& Row. 1968, $300 \mathrm{p}$.

(15) BARNES, B. V., K. S. PREGITZER, T. A. SPIES, V. H. SPOONER. Ecological forest site classification. Journal of Forestry, 1982, vol. 80, p. 493-498.

(16) WILLIS, B. El norte de Patagonia: naturaleza y riquezas. Buenos Aires: Comisión de Estudios Hidrológicos. Ministerio de Obras Públicas. 1914. 500 p.

(17) NÖBL, H. Documento fotográfico, planos de emplazamiento del centro de deportes invernales cerro Catedral y nota dirigida al director de Parques Nacionales Dr. Antonio Lynch. Buenos Aires. 1937.

(18) REBERTUS, A. J., T. T. VEBLEN. Structure and treefall gap of old-growth Nothofagus forests in Tierra del Fuego, Argentina. Journal of Vegetation Science, 1993 vol. 4, p. 641-654.

(19) GUTIERREZ, E., V. R. VALLEJO, J. ROMAÑA, J. FONS. The subantarctic Nothofagus forests of Tierra del Fuego: distribution, structure and production. Oecologia Aquatica, 1991, vol. 10, p. 351-366.

(20) PEARSON, A. K., O. P. PEARSON, I. A. GOMEZ Biology of the bamboo Chusquea culeou (Poaceae: Bambusoideae) in southern Argentina. Vegetatio, 1994 vol. 111 , p. $93-126$

(21) VAN REEUWIJK, L. P. ANDOSOLS. In: DRIESSEN P. M., R. DUDAL. The major soils of the world: lecture notes on their geography, formation, properties and use. Wageningen: Agricultural University / Katholieke Universiteit Leuven, 1991, p. 57-64.

(22) MORETTI A E. E. M. BESSERA, A A MARCOLIN. Plan de manejo para el ordenamiento territorial del área cerro Catedral: análisis preliminar de estabilidad estructural del medio físico. San Carlos de Bariloche Ministerio de Recursos Naturales de Río Negro, 1991, $53 \mathrm{p}$.

(23) UBEDA, C. A. D GRIGERA. Recalificación del estado de conservación de la fauna silvestre argentina. Región Patagónica. Buenos Aires: Secretaría de Recursos Naturales y Ambiente Humano. Consejo Asesor Regional Patagónico de la Fauna Silvestre. 1995. 95 p.

(24) PEET, R. K., N. L. CHRISTENSEN. Competition and tree death. BioScience, 1987, vol. 37, p. 586-595. 